\title{
The effect of the transition period and postpartum body weight loss on macrophage infiltrates in bovine subcutaneous adipose tissue
}

\author{
Ashleigh W. Newman, ${ }^{1}$ Andrew Miller, ${ }^{2}$ Francisco A. Leal Yepes, ${ }^{1}$ Elizabeth Bitsko, ${ }^{1}$ Daryl Nydam, ${ }^{1}$ \\ and Sabine Mann ${ }^{1 *}$ \\ ${ }^{1}$ Department of Population Medicine and Diagnostic Sciences, and \\ ${ }^{2}$ Department of Biomedical Sciences, Section of Anatomic Pathology, Cornell University, Ithaca, NY 14853
}

\begin{abstract}
The transition period in dairy cattle is characterized by a rapid increase in metabolic demands due to the onset of lactation in parallel with a voluntary drop in dry matter intake, which slowly increases compared with the rapidly increasing milk production. The resulting deficit in energy intake is largely met by lipolysis of adipose tissue stores liberating nonesterified fatty acids (NEFA) as energy substrates to support milk production and for the synthesis of milk fat. Previous work in cattle and other species has documented an increase in adipose tissue macrophages (ATM) during periods of feed restriction and lipolysis; however, alterations in ATM during the transition period have only recently received interest because of the role that adipose tissue remodeling and inflammation may play during this time. The primary objective of this study was to evaluate the number of macrophages in subcutaneous adipose tissue from dairy cattle at multiple time points throughout the transition period. Secondary objectives included the evaluation for potential associations of ATM with changes in body condition score (BCS) and concentrations of circulating NEFA and $\beta$-hydroxybutyrate. Holstein cows $(\mathrm{n}=25)$ were enrolled in the study $28 \mathrm{~d}$ before expected parturition and fed the same prepartum and postpartum rations for ad libitum consumption. On d 10 before expected parturition, and on d 4 and 21 after calving, subcutaneous adipose tissue biopsies were taken from the paralumbar fossa and preserved in formalin. Paraffinembedded sections of tissue from each of the 25 cows at the 3 time points (total of 75 samples) were labeled with an immunohistochemical marker of macrophages, ionized calcium-binding adapter molecule 1 (Iba1, also
\end{abstract}

Received July 10, 2018.

Accepted September 29, 2018.

*Corresponding author: sm682@cornell.edu known as allograft inflammatory factor 1). The number of Iba1-positive cells, the number of crown-like structures, and the number of immunopositive aggregates were enumerated in each sample. The number of Iba1positive macrophages increased by 60 and $93 \%$ on d 4 and 21 , respectively, when compared with the average prepartum number. Additionally, cows that lost $\geq 0.50$ points of BCS had a 57 and $52 \%$ higher number of ATM on d 4 and 21 postpartum, respectively, when compared with cows that lost $\leq 0.25$ points of BCS. No association was found between ATM numbers and NEFA and $\beta$-hydroxybutyrate concentrations or milk production in early lactation. Cows experiencing a more pronounced decrease in BCS postpartum had the largest increase in adipose tissue macrophage counts, suggesting that a greater degree of adipose tissue remodeling during the period of greatest nutrient deficit may be associated with higher numbers of ATM.

Key words: allograft inflammatory factor 1, ionized calcium-binding adapter molecule 1, inflammation, lipolysis, negative energy balance

\section{INTRODUCTION}

The transition period in dairy cattle is a physiologically challenging time due to the profound energy requirements associated with the initiation of lactation and the delayed increase in DMI to fulfill the nutrient needs. In addition, cows show a typical drop in feed intake in the peripartum period, and together this results in negative nutrient balance, which is most severe in the immediate postpartum period. To compensate for the lack in energy, lipolysis of triglyceride stores in adipose tissue (AT) occurs, releasing nonesterified fatty acids (NEFA) into circulation for tissue, particularly mammary gland, utilization. Excessive lipolysis is likely linked to the inflammatory dysfunction commonly observed in postpartum dairy cows undergoing metabolic stress (Sordillo and Raphael, 2013; Bradford et al., 2015), but the role of AT in this inflammatory response remains to be characterized. 
Much of the research on AT inflammation has been performed in mice with a focus on obesity and insulin resistance in humans (Hill et al., 2014). Here it has been established that lean AT contains low numbers of alternatively activated M2 resident macrophages involved in homeostatic functions, whereas obese AT contains high numbers of classically activated, proinflammatory M1 macrophages (Hill et al., 2014). The increase in adipose tissue macrophages (ATM) is likely multifactorial with lipolysis and adipocyte necrosis as 2 of the proposed drivers for the recruitment of ATM (Cinti et al., 2005; Kosteli et al., 2010). These ATM are proposed to have a protective function of scavenging excess local NEFA during periods of demand lipolysis based on the observations of intracellular lipid vacuoles in ATM and the increase in multinucleated lipid-laden macrophages (Cinti et al., 2005; Kosteli et al., 2010). In mice and humans, ATM have been observed to be arranged circumferentially around necrotic adipocytes in what has been termed crown-like structures (CLS; Cinti et al., 2005; Hill et al., 2014). These are rare in lean mice, but observed with increased frequency in obese mice (Cinti et al., 2005). The presence of CLS are evident in the results of a recent bovine study; however, they were not identified as such (Contreras et al., 2016). The question of the relevance of these findings in transition dairy cows is valid.

A recent study on ATM found minimal increases in early lactation cows (Akter et al., 2012). In response, the authors postulated that the level of fatness in the cows in their study may have been inadequate to stimulate significant ATM infiltrates (Akter et al., 2012). However, ATM infiltrates along with a milieu of proinflammatory cytokines were later demonstrated in ketotic transition dairy cattle with concurrent displacement of the abomasum (Contreras et al., 2015). This same group went on to research ATM in late-lactation dairy cattle given a brief 4 -d feed restriction (Contreras et al., 2016). Although a lipolytic drive and increase in ATM were documented, the authors acknowledged that their feed restriction model was unable to mimic the metabolic stress (e.g., hypoglycemia) experienced postpartum. Another group found low numbers of ATM in postpartum Holstein cows, implying macrophages play a minor role (Häussler et al., 2017). Most recently, the change in ATM throughout the transition period was studied (De Koster et al., 2018), and the authors found increased ATM in cows with high BCS loss supporting an association between ATM infiltration and body fat mobilization in early lactation dairy cows (De Koster et al., 2018). Given the dynamic and complex nature of the transition period, there is a need to study the change in ATM infiltrates in a larger cohort of dairy cattle at multiple times points throughout the transition period.

A variety of macrophage markers have been used to highlight ATM in the cow, which are restricted by the sample type available (e.g., fresh tissue in a fluid suspension, fresh frozen tissue/cryosection, or formalinfixed tissue). These markers include CD11b, CD11c, CD14, CD68, CD163, and CD172a (Akter et al., 2012; Contreras et al., 2015, 2016). These markers vary in their specificity for labeling cells of the monocyte/macrophage lineage. A relatively new and specific marker of macrophages is ionized calcium binding adaptor molecule 1 (Iba1), a calcium-binding protein involved in cell movement and phagocytosis (Imai et al., 1996; Ohsawa et al., 2000). The following aliases are used for Iba1: allograft inflammatory factor 1 (AIF-1, AIF1), interferon gamma responsive transcript (IRT-1, IRT1), balloon angioplasty responsive transcript 1 (BART-1, BART1), microglia response factor (MRF-1, MRF1), and protein G1 (G1). It was first discovered to be highly expressed in microglia (the resident macrophages of the brain; Ohsawa et al., 2000). Subsequently, Iba1 has been shown to be a histiocytic or "pan-macrophage" marker, as it is expressed by macrophages and dendritic cells in normal tissue, inflammatory conditions, and neoplasms (Köhler, 2007; Pierezan et al., 2014; Shimamoto et al., 2018). Importantly for research and diagnostic purposes, Iba1 can be used on formalin-fixed tissue.

Given the identified gaps in our knowledge of ATM in the bovine species, particularly those undergoing lipolysis, the primary objective of this study was to evaluate for the presence of macrophages in subcutaneous AT from dairy cattle at multiple time points throughout the transition period in relation to markers of lipolysis. We hypothesized that ATM infiltrates increase after calving. Secondary objectives included evaluation for potential associations of ATM with BCS, rate of AT lipolysis and loss of body condition, as well as concentrations of circulating metabolic markers. This study is also the first to report the use of Iba1 in bovine AT.

\section{MATERIALS AND METHODS}

\section{Animals and AT Samples}

Animals were enrolled in the study $28 \mathrm{~d}$ before expected parturition and housed in tiestalls with individual feed bins. All animals were fed the same prepartum and postpartum rations for ad libitum consumption as previously described (Mann et al., 2018). The analyzed composition of the dry and fresh period diet is shown in Table 1. Animals were eligible for enrollment if they 
Table 1. Analyzed composition (\% of DM unless otherwise noted; mean $\pm \mathrm{SD}$ ) of $\operatorname{diets}^{1}$

\begin{tabular}{lcc}
\hline Item & Dry & Fresh \\
\hline $\mathrm{DM}(\%)$ & $49.2 \pm 4.6$ & $52.0 \pm 2.9$ \\
$\mathrm{NE}_{\mathrm{L}}(\mathrm{Mcal} / \mathrm{kg}$ of $\mathrm{DM})$ & $1.48 \pm 0.05$ & $1.63 \pm 0.03$ \\
$\mathrm{CP}$ & $13.7 \pm 0.9$ & $15.5 \pm 0.8$ \\
$\mathrm{ADF}$ & $28.2 \pm 2.3$ & $21.6 \pm 1.6$ \\
$\mathrm{NDF}$ & $42.2 \pm 3.3$ & $31.7 \pm 2.1$ \\
$\mathrm{Starch}$ & $19.5 \pm 3.1$ & $25.7 \pm 2.7$ \\
Ether extract & $3.1 \pm 0.3$ & $3.4 \pm 0.2$ \\
$\mathrm{Ca}$ & $1.54 \pm 0.35$ & $0.89 \pm 0.09$ \\
$\mathrm{P}$ & $0.31 \pm 0.04$ & $0.36 \pm 0.03$ \\
$\mathrm{Mg}$ & $0.52 \pm 0.07$ & $0.46 \pm 0.05$ \\
$\mathrm{~K}$ & $1.20 \pm 0.16$ & $1.28 \pm 0.41$ \\
$\mathrm{DCAD}(\mathrm{mEq} / 100 \mathrm{~g}$ of $\mathrm{DM})$ & $-7.8 \pm 5.5$ & $31.7 \pm 6.1$ \\
\hline
\end{tabular}

${ }^{1}$ Near-infrared spectroscopy of 22 dry-period and fresh-period TMR composite samples, respectively (Cumberland Valley, Maugansville, MD).

were entering their second or greater lactation. On $d$ 10 before expected parturition, as well as on $\mathrm{d} 4$ and 21 after calving, subcutaneous AT samples were taken from the paralumbar fossa according to a technique described previously (Mann et al., 2016). Samples (approximately $0.5-0.8 \mathrm{~cm}^{3}$ sections) were blotted dry to remove excess blood and immediately submerged in buffered $10 \%$ formalin solution. Samples were fixed for at least $7 \mathrm{~d}$ in formalin, and subsequently embedded in paraffin blocks.

\section{Body Condition and Energy Metabolites}

Body condition was judged by one investigator at enrollment and weekly throughout the study according to a scale from 1 to 5 as described by Edmonson et al. (1989), and BW was determined weekly at the same time each day. Plasma BHB and NEFA concentrations were measured in samples 3 times per week immediately before the morning feeding at the same time every day using methods previously described (Mann et al., 2015; Leal Yepes et al., 2018). Hyperketonemia was defined as a blood BHB concentration of $\geq 1.2 \mathrm{mmol} / \mathrm{L}$ at least once between 3 to 21 DIM, and cows with high NEFA concentrations as those with $\geq 1 \mathrm{mmol} / \mathrm{L}$ in the same period. Cows were observed daily and disease events were recorded according to the farm standard protocol.

\section{Preparation of Samples for Histopathology and Immunohistochemical Staining Methodology}

Immunohistochemical staining (IHC) using an automated platform was performed (Bond-Max IHC/ISH platform, Bond polymer refine DAB kit; Leica Biosystems, Buffalo Grove, IL). Ionized calcium binding adap- tor molecule 1 (Wako Chemical, Richmond, VA) was used to identify macrophages. Before use of Iba1 in this study, the IHC marker was validated for use in cattle. The Iba1 was applied to a bovine tissue microarray consisting of brain, kidney, liver, small intestine, lymph node, lung, liver, heart, skeletal muscle, and spleen. Validation of Iba1 was based on the immunoreactivity of the antibody with macrophage-lineage cells in an expected anatomic distribution in all tissues. Bovine lymph node was used as the control tissue. The negative control tissue was an isotype matched secondary antibody. The positive and negative controls stained as expected. Representative images of Iba1 staining in the tissue microarray, including the positive and negative controls, are provided in Supplemental Figure S1 (https://doi.org/10.3168/jds.2018-15362).

Unstained, paraffin-embedded sections cut at $5 \mu \mathrm{m}$ were dewaxed with the Bond dewax solution (Leica Biosystems) followed by a heat epitope retrieval with Bond epitope retrieval system for 10 min (Leica Biosystems). This was followed by a 5 -min block with $3 \%$ hydrogen peroxide (Leica Biosystems). The Iba1 antibody was applied for $15 \mathrm{~min}$ at a dilution of 1:3,000. This was followed by Leica Bond Polymer refine detection for 10 min. The $3^{\prime} 5^{\prime}$-diaminobenzidine was applied for $5 \mathrm{~min}$ (Leica Biosystems). Last, slides were counterstained with hematoxylin (Sigma-Aldrich, St. Louis, MO) for 5 min.

The IHC slides were scored by a single investigator blinded to sample ID and time point to avoid bias during scoring. To achieve this, slides were arranged randomly in blocks of 3 cows using an open-access web-based randomizer (Urbaniak and Plous, 2012). Slides were scored twice in 2 independent sessions, and the average count between the 2 results was used for analysis. Each slide was first examined at low power (10× objective) to observe the overall histological appearance of the AT sample. The Iba1-positive cells were counted in 10 random fields using the $40 \times$ objective. The Iba1-positive cells were defined as a cell with a visible nucleus and positive Iba1 cytoplasmic staining.

A CLS was defined as multiple Iba1-positive cells arranged circumferentially around a clear space (presumptive adipocyte; Figure 1A). An aggregate was defined as multiple Iba1-positive cells arranged in a solid group, with no central clear space (Figure 1B and 1C). A multinucleated giant cell was defined as a large mass of positively staining cytoplasm containing multiple nuclei (Figure 1D). The CLS and macrophage aggregates were enumerated in one low power field ( $4 \times$ objective) on each of the Iba1 IHC slides. The samples were also evaluated for the presence of multinucleated giant cells. The slides were enumerated in the same random blocks 


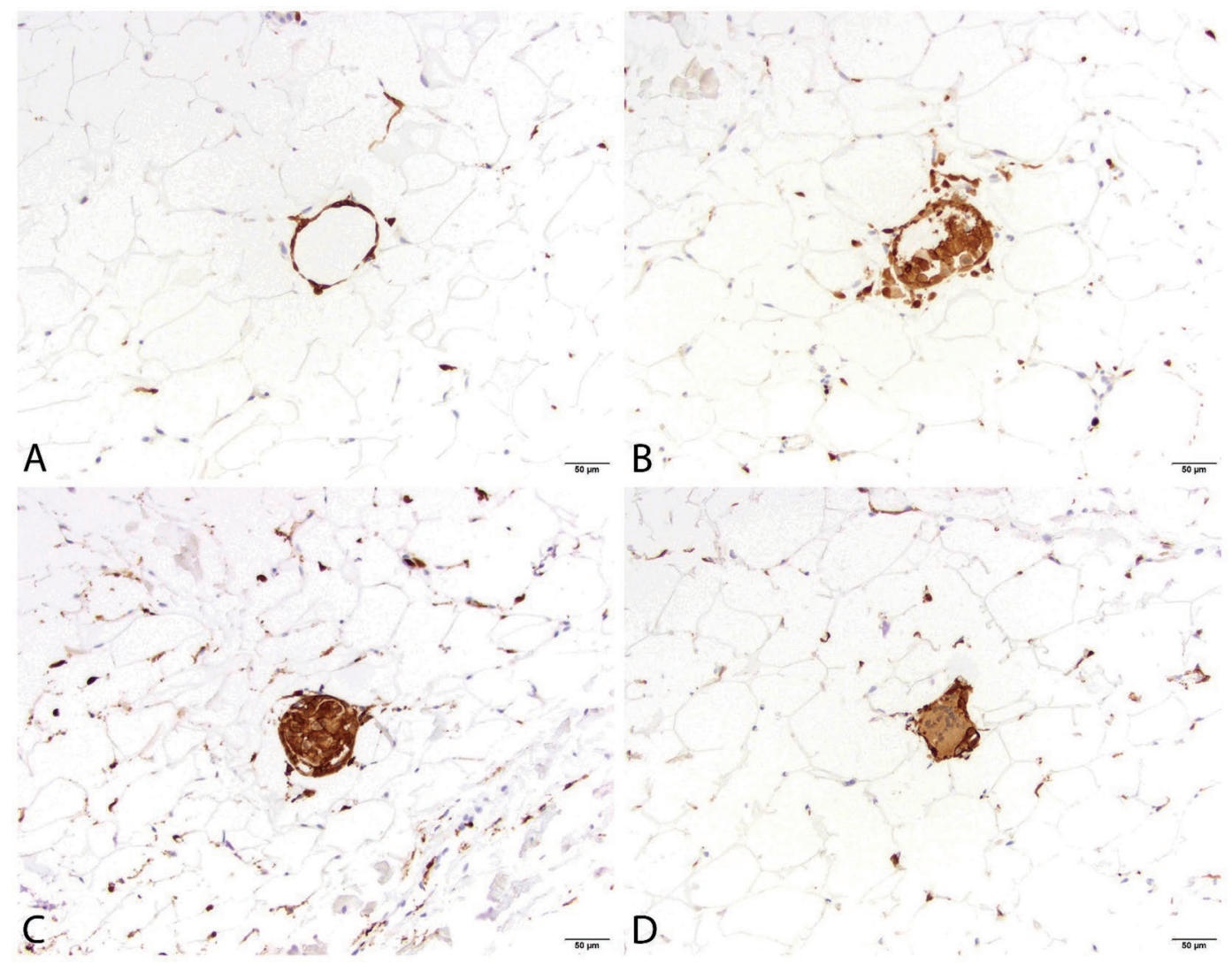

Figure 1. Photomicrographs of subcutaneous adipose tissue samples, stained with ionized calcium binding adaptor molecule 1 (Iba1), from 4 different cows. Macrophages stain brown with Iba1. (A) Example of a crown-like structure (CLS) from a cow 4 d postpartum. One CLS was defined as multiple Iba1-positive cells arranged circumferentially around a clear space (presumptive adipocyte). (B) Depicts what appears to be a CLS that has collapsed and progressed to digestion of the adipocyte. This sample was from a cow $4 \mathrm{~d}$ postpartum. (C) Example of an aggregate from a cow $21 \mathrm{~d}$ postpartum. One aggregate was defined as multiple Iba1-positive cells arranged in a solid group, with no central clear space (B and $\mathrm{C}$ would both be counted as aggregates). (D) Example of a multinucleated giant cell, which likely represents the fusion of an aggregate of macrophages. This was from a cow $4 \mathrm{~d}$ postpartum (Iba1, $20 \times$ objective, scale bar $=50 \mu \mathrm{m}$ ).

of 3 cows and total number of CLS and macrophage aggregates were documented.

\section{Analytical Approach}

The main objective was to describe the change in macrophage numbers in AT samples over time in this convenience sample of 25 animals. To analyze this, the average number of Iba1-positive cells in 2 sets of 10 random $40 \times$ microscopic fields was considered the primary outcome. A repeated measures model was fit to the data using Proc MIXED with time as the fixed as well as repeated effect and cow as the subject. Parity dichotomized in first versus greater lactation was included as a covariate. Given the unevenly spaced distribution of observations, unstructured and 3 spatial (Gaussian, power, and spherical) covariance structures were tested and the one with the lowest Akaike information criterion was chosen for the final model fit. To achieve normality and homoscedasticity of residuals, the average Iba1 count was log-transformed.

The secondary objectives included associations of macrophage numbers in AT with average body condition in the peripartum period, body condition loss, BHB, and NEFA concentrations on a continuous scale, as well as with the dichotomous variables postpartum hyperketonemia (HYK/non-HYK) and increased NEFA concentrations (high NEFA/low NEFA). Correlations of the count of Iba1-positive cells at each time point with BCS, BHB, and NEFA concentrations were assessed with Spearman's $\rho$. Body condition loss was dichotomized in 2 categories: cows that lost $0.25 \mathrm{BCS}$ points and less, and those that lost 0.5 points and more from calving to wk 6 postpartum. To test associations with these measurements, each independent variable was tested in the model described above and the same covariance structures were tested. All parameters that were found to be associated with macrophage numbers 


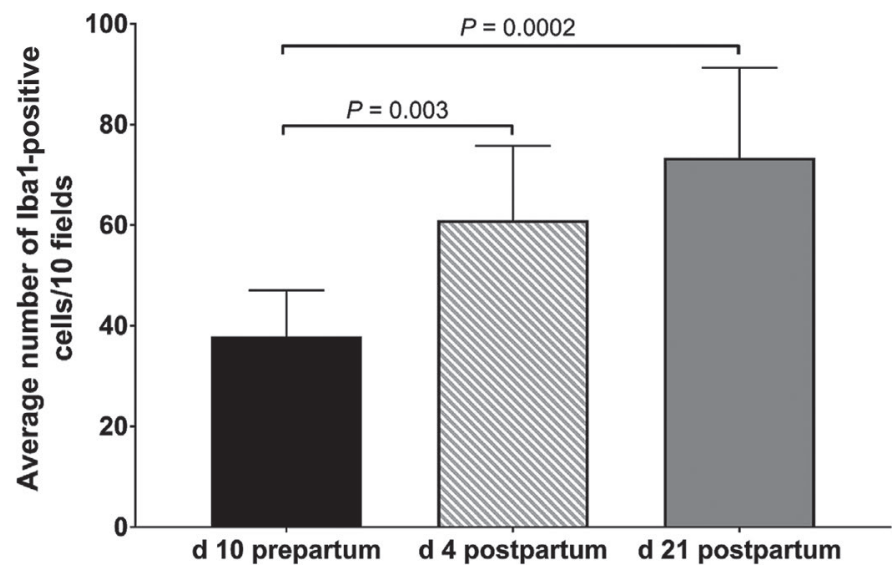

Figure 2. Geometric LSM and 95\% CI of the average number of ionized calcium binding adaptor molecule 1 (Iba1)-positive cells per 10 high-power fields (40× objective) in subcutaneous adipose tissue samples of 25 Holstein dairy cows at $10 \mathrm{~d}$ prepartum, as well as 4 and $21 \mathrm{~d}$ postpartum. The $P$-values of repeated measures ANOVA for the fixed effect of time was corrected for all pairwise comparisons using Tukey's procedure.

were then tested together in one model to test possible interactions.

Number of aggregates and CLS were compared for different sampling time points using Wilcoxon tests (Proc NPAR1WAY) because data distribution was found to be nonparametric.

All results are presented as mean or geometric mean and $95 \%$ CI unless otherwise stated.

\section{RESULTS}

\section{Description of Study Population}

Adipose tissue samples from a convenience sample of 25 animals were included in the study. At enrollment, $14,6,4$, and 1 animal were in lactation $1,2,3$, and 4 , respectively. The average (95\% CI) sampling day relative to calving was -10.3 ( -8.0 to -12.6$), 4.4$ (4.1 to 4.7), and 20.1 (19.6 to 20.6). At enrollment, 13, 8, and 4 animals had a BCS of 3, 3.25, and 3.5, respectively. The average weight in the first week postpartum was 740 (712 to 769$) \mathrm{kg}$, and cows produced an average of 38.1 (32.9 to 43.8$) \mathrm{kg} / \mathrm{d}$ of milk in the first 21 DIM.

\section{Description of Macrophage Infiltrates in Adipose Tissue in the Peripartum Period}

The number of Iba1-positive cells increased over time with both postpartum time points showing higher results compared with the prepartum sample $(P=0.003$ and $P=0.0002$, respectively; Figure 2). Figure 3 shows samples from one cow with a typical increase in macrophages in AT over time. The number of CLS in each of the samples is shown in Figure 4. The number of CLS per tissue sample showed an increase from the prepartum to the postpartum samples, but overall differences over time were not conclusive $(P=0.13)$. The number of aggregates was not different over time $(P=0.57)$. When summing the number of CLS and aggregates for each sample, nonparametric analysis showed no difference over time $(P=0.26)$. Multinucleated giant cells were rarely noted in both prepartum and postpartum tissue samples.

\section{Association of Macrophage Numbers with Body Condition and Metabolic Markers}

Spearman's $\rho$ correlation at each of the 3 time points with BCS and metabolic markers yielded only weak correlations $(\rho<0.40)$. The strongest correlation was observed between Iba1 counts on d 4 postpartum with counts on d 21 postpartum, whereas both postpartum time points showed no correlation with the prepartum

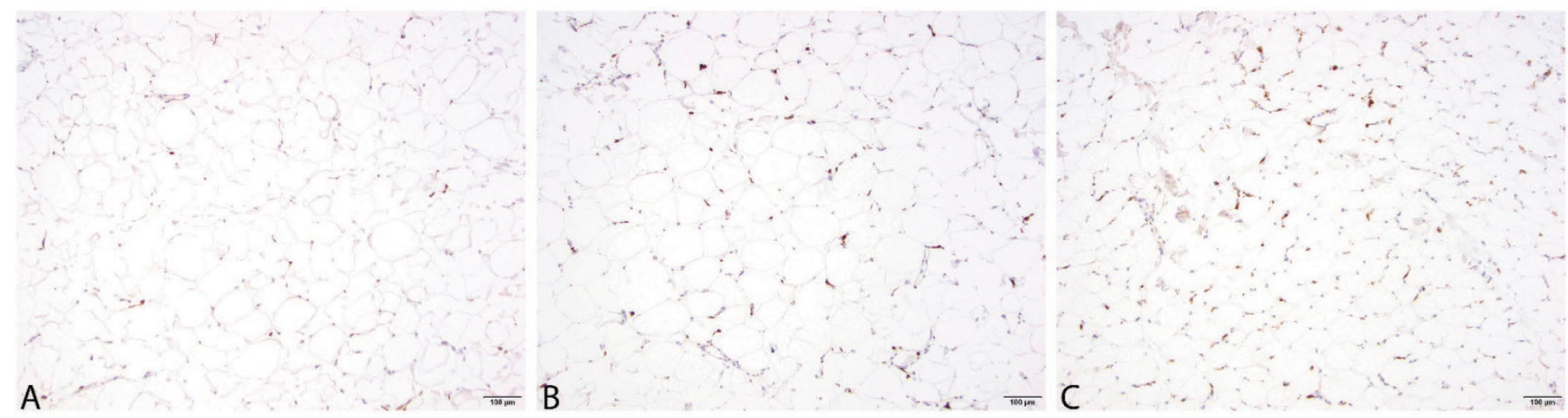

Figure 3. Photomicrographs of subcutaneous adipose tissue samples from one cow across each of the sampled time points: $10 \mathrm{~d}$ prepartum (A), 4 d postpartum (B), and 21 d postpartum (C). Ionized calcium binding adaptor molecule 1 (Iba1)-positive cells (macrophages) are brown in color (Iba1, $10 \times$ objective). 


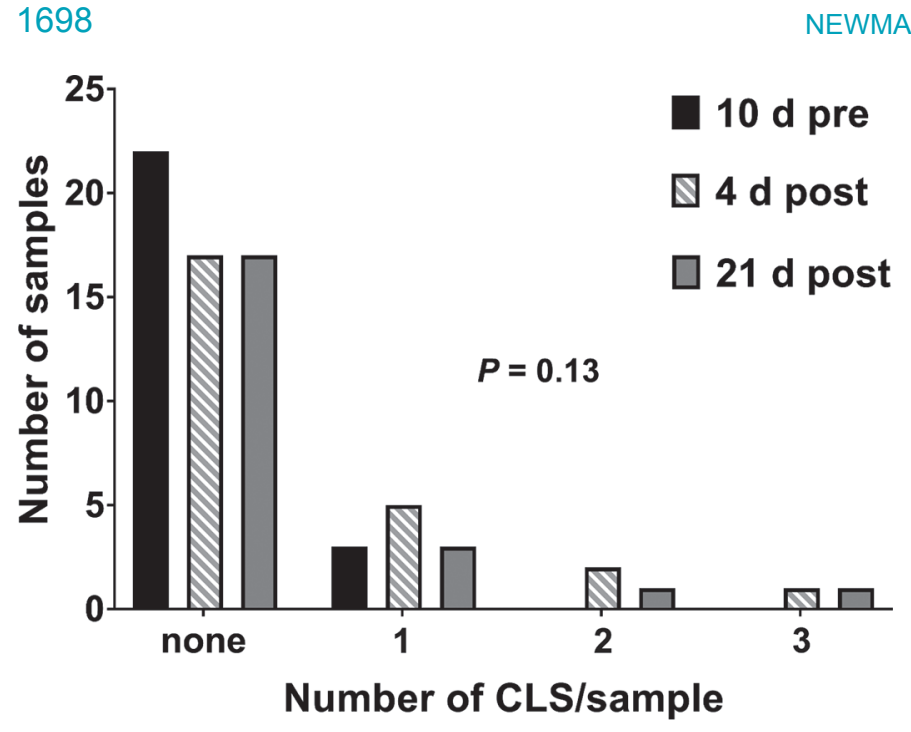

Figure 4. Number of crown-like structures (CLS) in each of the 25 samples per sampling time point pre- and postpartum. One CLS was defined as multiple ionized calcium binding adaptor molecule 1 (Iba1)-positive cells arranged circumferentially around a clear space (presumptive adipocyte). The $P$-value for the difference over time was generated using Wilcoxon tests.

one. The average peripartum BCS showed little variation in the sample population of 25 animals (median 3.05 ; range 2.80 to 3.40 ) and was not associated with the average number of Iba1-positive cells in the AT samples $(P=0.56)$. Eight $(32 \%), 7(28 \%)$, and $1(4 \%)$ cow lost $0.25,0.5$, and 0.75 BCS points, respectively, whereas $8(32 \%)$ cows showed no BCS loss over the

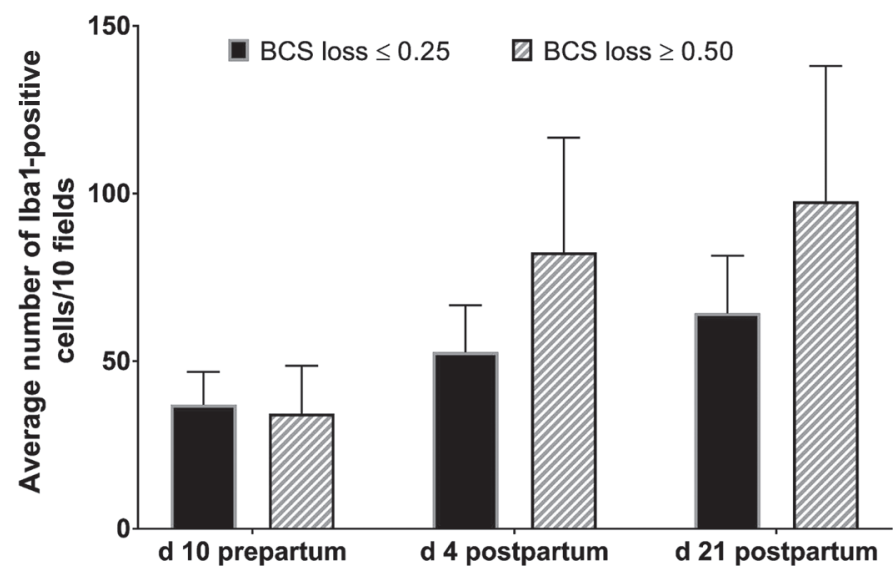

Figure 5. Geometric LSM and 95\% CI of the average number of Iba1-positive cells per 10 high-power fields ( $40 \times$ objective) in subcutaneous adipose tissue samples of 25 Holstein dairy cows at 3 different time points in the transition period stratified by BCS loss $(\leq 0.25$ vs. $\geq 0.50$ BCS points). Time: $P<0.0001$, BCS loss: $P=0.05$, time $\times$ BCS loss: $P=0.13$. Iba1 $=$ ionized calcium binding adaptor molecule 1. first 6 wk postpartum, and $1(4 \%)$ cow gained 0.25 points of BCS. Cows that lost $\geq 0.5$ points of BCS had a higher number of macrophages in AT samples (Figure $5)$, whereas no association was found with BW loss ( $P$ $=0.36)$. Milk production was not associated with macrophage numbers $(P=0.49)$.

The average concentration of NEFA and BHB for each cow calculated from all samples in the first 21 DIM showed individual variation (NEFA: median 0.55 , range 0.33 to $1.16 \mathrm{mmol} / \mathrm{L}$; BHB: median 1.00, range 0.67 to $2.51 \mathrm{mmol} / \mathrm{L})$. Thirteen cows $(52 \%)$ had a plasma NEFA concentration above $1 \mathrm{mmol} / \mathrm{L}$ at least once in the same period and were classified as "high NEFA," but this was not associated with the average macrophage count in AT $(P=0.54)$. Fifteen cows $(60 \%)$ had a whole-blood $\mathrm{BHB}$ concentration $\geq 1.2 \mathrm{mmol} / \mathrm{L}$ at least once between 3 to 21 DIM and were classified as hyperketonemic, but this was also not associated with the average macrophage count $(P=0.45)$.

Seventeen animals $(68 \%)$ remained healthy in the first 21 DIM, whereas 8 animals (32\%) showed at least one postpartum disorder, including 2 cases of milk fever, 5 cases of retention of the fetal membranes, 2 cases of pneumonia, and one case of clinical mastitis. Due to the small number of cows enrolled in this study and the low frequency of health events in each category, no further analysis was performed.

\section{DISCUSSION}

This study characterized the change in ATM in dairy cattle at multiple time points throughout the transition period. This is also the first study utilizing Iba1 to label ATM in cattle. Concordant with our hypothesis, a higher number of ATM was present postpartum as compared with prepartum. Additionally, greater postpartum BCS loss was associated with a higher number of ATM in this study population.

Macrophages are important for both tissue repair (M2 macrophages), as well as mounting an inflammatory response (M1 macrophages) with the liberation of pro-inflammatory cytokines (e.g., TNFo and IL6) triggering the acute phase response (Sordillo and Raphael, 2013; Kumar et al., 2015). The increase in ATM postpartum supports the concept that parturition and thus the transition period is associated with inflammatory processes and tissue remodeling. Inflammation, while often carrying a negative connotation, can be appropriate and adaptive returning the body back to homeostasis (Bradford et al., 2015). Disease results when inflammation is excessive and prolonged. As demonstrated in this study, Iba1 is a reliable IHC marker of macrophages; however, a limitation is its 
labeling of both M1 and M2 macrophages (Pan et al., 2015). Therefore, we were unable to distinguish the nature of the ATM infiltrate in this study. However, previous work in this field has identified gene expression profiles in AT consistent with an M1/inflammatory process (Contreras et al., 2015; De Koster et al., 2018). The expression of CCL2, also known as macrophage chemotactic protein-1, and CCL22, also known as macrophage-derived chemoattractant, support the notion that macrophages are recruited to the AT as an inflammatory infiltrate (Contreras et al., 2015; De Koster et al., 2018). Additionally, a very low mitotic rate in the AT of fasted mice supports ATM are an infiltrative population, rather than the result of a proliferative process within the tissue (Kosteli et al., 2010).

Crown-like structures and multinucleated giant cells were identified in this study, as has been reported in the human and murine literature. Crown-like structures designate sites of adipocyte death, which are believed to form as a protective mechanism to scavenge free lipid (Cinti et al., 2005). Cinti et al. (2005) confirmed the adipocytes as necrotic on the basis of electron microscopy and the absence of immunoreactivity for the essential lipid droplet-associated protein perilipin, which was present in viable adipocytes. Although this additional testing was not performed in the current study, we are confident that what we identified as CLS share the classic histologic appearance demonstrated by this group and others (Kosteli et al., 2010). The digestion of necrotic adipocytes is consistent with one of the functions of macrophages to eliminate dead cellular debris (Kumar et al., 2015). Inflammation involving AT (e.g., panniculitis) is frequently granulomatous consisting of multinucleated giant cells, which form as a result of fusion of 2 or more activated macrophages (O'Kell et al., 2010; Kumar et al., 2015). Therefore, it is believed that CLS ultimately progress to form multinucleated giant cells (Cinti et al., 2005). We support this notion, as documented in our results, and propose that following CLS formation, it collapses as the adipocyte is digested leading to an aggregate of macrophages, and ultimately a multinucleated giant cell. Interestingly, multinucleated giant cells have been reported in obese, but not lean, mice and humans (Cinti et al., 2005).

Approximately one-third of cows lost $>0.5$ BCS points throughout the course of the study, which was associated with a higher number of ATM postpartum. This drop in BCS can be equated to a decrease in BW, particularly AT loss, due to negative nutrient balance during the transition period. It was surprising that no association with BW loss itself was observed. This could be explained by the fact that BW loss or gain can be due to other tissues in addition to AT, such as the mammary gland, gastrointestinal tract, and changes in lean body mass. The change in BCS, on the other hand, allowed us to specifically describe changes in subcutaneously located AT mass, which correlates moderately well with abdominal fat mass.

Our results agree with the finding of increased ATM in the early period $(0-7 \mathrm{~d})$ of weight loss in lean mice, supporting the idea of lipolysis and adipocyte death as a signal for macrophage recruitment to AT (Kosteli et al., 2010). In that study, the increase was attributed to an infiltrate of anti-inflammatory $\left(\mathrm{CD} 11 \mathrm{c}^{-}\right)$population of macrophages and an absence of a pro-inflammatory gene signature (Kosteli et al., 2010; Red Eagle and Chawla, 2010). Similarly, in a weight loss study in obese postmenopausal women, an increase in CLS density was documented without a corresponding increase in pro-inflammatory genes in AT (Alemán et al., 2017). Therefore, although the presence of ATM, CLS, and multinucleated giant cells may suggest pathologic inflammation, we support the concept that ATM are a physiologic response to lipolysis functioning to remodel AT and prevent local lipotoxicity. Additionally, the finding of a decrease in ATM with prolonged weight loss ( $>21$ d) in mice supports that ATM associated with lipolysis does not proceed unchecked, resulting in chronic inflammation (Kosteli et al., 2010; Red Eagle and Chawla, 2010).

In these weight loss studies in mice and women, an association has been observed between weight loss, ATM, and circulating NEFA and BHB levels (Kosteli et al., 2010; Red Eagle and Chawla, 2010; Alemán et al., 2017). In our study, despite more than half of the cows having high NEFA or BHB results, no association was observed with these outcomes and ATM numbers, including CLS and aggregates. We do not believe our results disprove the connection between lipolysis and ATM, but rather suggests that differences in the use of fatty acids from lipolysis exist. In the mice and women, caloric restriction was used as a means to induce weight loss (Kosteli et al., 2010; Alemán et al., 2017) without additional means to increase fatty acid usage, for example through adjunctive physical exercise. However, in postpartum dairy cattle, a large drain of fatty acids occurs from the bloodstream due to the use of fatty acids as alternative energy sources as well as directly for milk fat synthesis in the mammary gland. Therefore, an association of ATM and NEFA or BHB may not have been detected in our study, due to the cows' efficient and physiological utilization of these energy building blocks in the liver and, particularly, the mammary gland. An alternative explanation is that the convenience sample of 25 animals may have been insufficient to detect associations if indeed these exist. However, to our knowledge, this is the largest study sampling cows at multiple time points to date. 
Another potential explanation for the lack of association between markers of negative nutrient balance and ATM is the location of AT samples obtained in this study. The majority of ATM in humans accumulate in omental, rather than subcutaneous adipose depots (Hill et al., 2014). Cattle also have slightly lower ATM infiltrates in subcutaneous as compared with visceral fat (Akter et al., 2012). However, the weight loss studies in mice and women both sampled subcutaneous AT (Kosteli et al., 2010; Alemán et al., 2017). The evaluation of visceral AT samples might have yielded different results regarding the association with ATM.

Measurement of adipocyte size or enumeration of ATM in relation to the number of adipocytes has been done in previous studies. However, adipocyte size can change with the processing of tissue into histologic sections, resulting in a questionable accuracy of those measurements (Laforest et al., 2017). Due to the inherent inconsistencies in adipocyte size due to fixation, we chose to enumerate the number of cells in an equivalent number of fields across all cases. This allowed us to perform the same standard measurement in each case, and the increased number of fields reviewed should decrease the risk for observer error.

The absence of an association between the number of CLS and any of the evaluated outcomes except rapid BCS loss may be due to the narrow range of BCS of the cows in this study. As Cinti et al. (2005) documented, CLS are more prevalent in obese individuals. Therefore, the absence of obesity in well-managed, high-producing dairy cattle likely limited our ability to detect an association if it exists.

\section{CONCLUSIONS}

We conclude that ATM, as successfully identified with the IHC marker Iba1, are present in higher numbers postpartum and associated with a greater degree of postpartum BCS loss. The ATM infiltrates reflect lipolysis and local AT remodeling in the postpartum cow.

\section{ACKNOWLEDGMENTS}

This work was supported by the USDA National Institute of Food and Agriculture (NIFA, Washington, DC; Hatch project 1007477). Any opinions, findings, conclusions, or recommendations expressed in this publication are those of the author(s) and do not necessarily reflect the views of NIFA or the United States Department of Agriculture (USDA). We thank Pharmadoc, Lüdersdorg, Germany, and TaiDoc, Taiwan, for the donation of BHB meters and strips used in this study. The authors thank Charlene Ryan (Cornell University,
Ithaca, NY) for her invaluable assistance with data collection. We also thank the staff at the research dairy for the care of the animals used in this study.

\section{REFERENCES}

Akter, S. H., S. Haussler, D. Germeroth, D. Von Soosten, S. Danicke, K. H. Sudekum, and H. Sauerwein. 2012. Immunohistochemical characterization of phagocytic immune cell infiltration into different adipose tissue depots of dairy cows during early lactation. J. Dairy Sci. 95:3032-3044. https://doi.org/10.3168/jds.2011-4856.

Alemán, J. O., N. M. Iyengar, J. M. Walker, G. L. Milne, J. C. Da Rosa, Y. Liang, D. D. Giri, X. K. Zhou, M. N. Pollak, C. A. Hudis, J. L. Breslow, P. R. Holt, and A. J. Dannenberg. 2017. Effects of rapid weight loss on systemic and adipose tissue inflammation and metabolism in obese postmenopausal women. J. Endocrinol. Soc. 1:625-637. https://doi.org/10.1210/js.2017-00020.

Bradford, B. J., K. Yuan, J. K. Farney, L. K. Mamedova, and A. J. Carpenter. 2015. Invited review: Inflammation during the transition to lactation: New adventures with an old flame. J. Dairy Sci. 98:6631-6650. https://doi.org/10.3168/jds.2015-9683.

Cinti, S., G. Mitchell, G. Barbatelli, I. Murano, E. Ceresi, E. Faloia, S. Wang, M. Fortier, A. S. Greenberg, and M. S. Obin. 2005. Adipocyte death defines macrophage localization and function in adipose tissue of obese mice and humans. J. Lipid Res. 46:2347-2355. https://doi.org/10.1194/jlr.M500294-JLR200.

Contreras, G. A., E. Kabara, J. Brester, L. Neuder, and M. Kiupel. 2015. Macrophage infiltration in the omental and subcutaneous adipose tissues of dairy cows with displaced abomasum. J. Dairy Sci. 98:6176-6187. https://doi.org/10.3168/jds.2015-9370.

Contreras, G. A., K. Thelen, S. E. Schmidt, C. Strieder-Barboza, C. L. Preseault, W. Raphael, M. Kiupel, J. Caron, and A. L. Lock. 2016. Adipose tissue remodeling in late-lactation dairy cows during feed-restriction-induced negative energy balance. J. Dairy Sci. 99:10009-10021. https://doi.org/10.3168/jds.2016-11552.

De Koster, J., C. Strieder-Barboza, J. de Souza, A. L. Lock, and G. A. Contreras. 2018. Short communication: Effects of body fat mobilization on macrophage infiltration in adipose tissue of early lactation dairy cows. J. Dairy Sci. 101:7608-7613. https://doi.org/10 $.3168 /$ jds.2017-14318.

Edmonson, A. J., I. J. Lean, L. D. Weaver, T. Farver, and G. Webster. 1989. A body condition scoring chart for Holstein dairy cows. J. Dairy Sci. 72:68-78. https://doi.org/10.3168/jds.2016-10969.

Häussler, S., D. Germeroth, L. Laubenthal, L. F. Ruda, J. Rehage, S. Dänicke, and H. Sauerwein. 2017. Short communication: Immunohistochemical localization of the immune cell marker CD68 in bovine adipose tissue: Impact of tissue alterations and excessive fat accumulation in dairy cows. Vet. Immunol. Immunopathol. 183:45-48. https://doi.org/10.1016/j.vetimm.2016.12.005.

Hill, A. A., W. Reid Bolus, and A. H. Hasty. 2014. A decade of progress in adipose tissue macrophage biology. Immunol. Rev. 262:134152. https://doi.org/10.1111/imr.12216.

Imai, Y., I. Ibata, D. Ito, K. Ohsawa, and S. Kohsaka. 1996. A novel gene iba1 in the major histocompatibility complex class III region encoding an EF hand protein expressed in a monocytic lineage. Biochem. Biophys. Res. Commun. 224:855-862. https://doi.org/ 10.1006/bbrc.1996.1112

Köhler, C. 2007. Allograft inflammatory factor-1/Ionized calciumbinding adapter molecule 1 is specifically expressed by most subpopulations of macrophages and spermatids in testis. Cell Tissue Res. 330:291-302. https://doi.org/10.1007/s00441-007-0474-7.

Kosteli, A., E. Sugaru, G. Haemmerle, J. F. Martin, J. Lei, R. Zechner, and A. W. Ferrante. 2010. Weight loss and lipolysis promote a dynamic immune response in murine adipose tissue. J. Clin. Invest. 120:3466-3479. https://doi.org/10.1172/JCI42845.

Kumar, V., A. K. Abbas, and J. C. Aster. 2015. Robbins and Cotran Pathologic Basis of Disease. 9th ed. Elsevier Saunders, Philadelphia, PA.

Laforest, S., A. Michaud, G. Paris, M. Pelletier, H. Vidal, A. Géloën, and A. Tchernof. 2017. Comparative analysis of three human 
adipocyte size measurement methods and their relevance for cardiometabolic risk. Obesity (Silver Spring) 25:122-131. https://doi .org/10.1002/oby.21697.

Leal Yepes, F. A. L., D. V. Nydam, W. Heuwieser, and S. Mann. 2018. Technical note: Evaluation of the diagnostic accuracy of 2 pointof-care $\beta$-hydroxybutyrate devices in stored bovine plasma at room temperature and at $37^{\circ} \mathrm{C}$. J. Dairy Sci. 101:6455-6461. https://doi .org/10.3168/jds.2017-13960.

Mann, S., F. A. Leal Yepes, T. R. Overton, J. J. Wakshlag, A. L. Lock, C. M. Ryan, and D. V. Nydam. 2015. Dry period plane of energy: Effects on feed intake, energy balance, milk production, and composition in transition dairy cows. J. Dairy Sci. 98:3366-3382. https://doi.org/10.3168/jds.2014-9024.

Mann, S., D. V. Nydam, A. Abuelo, F. A. Leal Yepes, T. R. Overton, and J. J. Wakshlag. 2016. Insulin signaling, inflammation, and lipolysis in subcutaneous adipose tissue of transition dairy cows either overfed energy during the prepartum period or fed a controlled-energy diet. J. Dairy Sci. 99:6737-6752. https://doi.org/10 .3168/jds.2016-10969

Mann, S., A. Sipka, F. A. Leal Yepes, D. V. Nydam, T. R. Overton, and J. J. Wakshlag. 2018. Nutrient-sensing kinase signaling in bovine immune cells is altered during the postpartum nutrient deficit: A possible role in transition cow inflammatory response. J. Dairy Sci. 101:9360-9370. https://doi.org/10.3168/jds.2018-14549.

Ohsawa, K., Y. Imai, H. Kanazawa, Y. Sasaki, and S. Kohsaka. 2000 Involvement of Iba1 in membrane ruffling and phagocytosis of macrophages/microglia. J. Cell Sci. 113(Pt 1):3073-3084.

O'Kell, A. L., N. Inteeworn, S. F. Diaz, G. K. Saunders, and D. L. Panciera. 2010. Canine sterile nodular panniculitis: A retrospective study of 14 cases. J. Vet. Intern. Med. 24:278-284.
Pan, J., J.-L. Jin, H.-M. Ge, K.-L. Yin, X. Chen, L.-J. Han, Y. Chen, L. Qian, X.-X. Li, and Y. Xu. 2015. Malibatol A regulates microglia M1/M2 polarization in experimental stroke in a PPAR $\gamma$ dependent manner. J. Neuroinflammation 12:51. https://doi.org/ 10.1186/s12974-015-0270-3.

Pierezan, F., J. Mansell, A. Ambrus, and A. R. Hoffmann. 2014. Immunohistochemical expression of ionized calcium binding adapter molecule 1 in cutaneous histiocytic proliferative, neoplastic and inflammatory disorders of dogs and cats. J. Comp. Pathol. 151:347351. https://doi.org/10.1016/j.jcpa.2014.07.003.

Red Eagle, A., and A. Chawla. 2010. In obesity and weight loss, all roads lead to the mighty macrophage. J. Clin. Invest. 120:34373440. https://doi.org/10.1172/JCI44721.

Shimamoto, Y., J. Nio-Kobayashi, H. Watarai, M. Nagano, N. Saito, E. Takahashi, H. Higuchi, A. Kobayashi, T. Kimura, and H. Kitamura. 2018. Generation and validation of novel anti-bovine CD163 monoclonal antibodies ABM-1A9 and ABM-2D6. Vet. Immunol. Immunopathol. 198:6-13. https://doi.org/10.1016/j.vetimm.2018 .02 .004 .

Sordillo, L. M. and W. Raphael. 2013. Significance of metabolic stress, lipid mobilization, and inflammation on transition cow disorders. Vet. Clin. North Am. Food Anim. Pract. 29:267-278. https://doi .org/10.1016/j.cvfa.2013.03.002.

Urbaniak, G. C., and S. Plous. 2012 Research Randomizer version 4.0 Accessed Oct. 10, 2017. http://www.randomizer.org/form.htm. 